Crítica de libro:

\title{
CLEMENTE PENALVA VERDÚ, ANTONIO ALAMINOS CHICA, FRANCISCO JOSÉ FRANCÉS GARCÍA Y ÓSCAR ANTONIO SANTACREU FERNÁNDEZ \\ LA INVESTIGACIÓN CUALITATIVA: TÉCNICAS DE INVESTIGACIÓN Y ANÁLISIS CON ATLAS.TI
}

Cuenca (Ecuador): PYDLOS Ediciones, 2015

\author{
Agustín García-Ramos \\ Universidad de Alicante, España \\ agusgarciaramos@gmail.com
}

La investigación cualitativa: técnicas de investigación y análisis con Atlas.ti forma parte de una serie de cinco monografías, aparecidas gracias a la estrecha colaboración docente e investigadora que durante los últimos años vienen manteniendo el Programa Interdisciplinario de Población y Desarrollo Local Sustentable (PYDLOS), de la Universidad de Cuenca (Ecuador), y el Observatorio Europeo de Tendencias Sociales (OBETS), integrado en el Instituto Interuniversitario de Desarrollo Social y Paz de la Universidad de Alicante. El primero de ellos nace en 1993 de la transformación del Centro de Estudios de Población y Desarrollo (CEPD) a raíz del conocido como "Desastre de la Josefina" -una de las mayores crisis socio-ambientales acaecidas en Sudamérica durante el siglo pasado- y se vincula a la formación de talentos humanos, la producción, sistematización y análisis de información en los ámbitos de población, territorio y desarrollo, así como al acompañamiento a procesos de planificación locales y regionales bajo el enfoque del Buen Vivir y el pensamiento crítico. Por su parte, el grupo de trabajo de OBETS, con una vocación marcadamente interdisciplinar, centra su interés en estudios de diagnóstico y prospectiva de carácter intercultural, económico, cívico y político sobre los comportamientos, actitudes y valores en el marco de referencia de la Unión Europea. 
Fruto de la citada colaboración entre las dos instituciones se están desarrollando, en el momento de escribir estas líneas, diversas vías de cooperación, una de las cuales ha cristalizado en el curso de formación de posgrado "Investigación Aplicada en Ciencias Sociales: Técnicas de producción de datos y análisis", que se imparte en la Universidad de Cuenca desde 2014. Este consta de cinco bloques, para cuyo desarrollo, tal como se anticipaba al inicio del presente escrito, se han elaborado, desde la Universidad de Alicante, otras tantas monografías -que, dicho sea de paso, son de libre acceso y pueden descargarse gratuitamente desde los repositorios institucionales de ambas instituciones educativas-. En concreto, los textos de la obra que nos ocupa se han preparado como referencia del bloque IV, intitulado "La perspectiva cualitativa. Análisis de datos textuales". Completan la lista Introducción a los modelos estructurales en investigación social, La investigación participativa: métodos y técnicas, Introducción a los modelos estructurales en investigación social y, por último, Análisis multivariante para las Ciencias Sociales I. Índices de distancia, conglomerados y análisis factorial, todos ellos desarrollados al alimón por los investigadores del ya aludido Instituto Interuniversitario de Desarrollo Social y Paz, a la vez que profesores adscritos al Departamento de Sociología II de la Universidad de Alicante, Antonio Alaminos Chica, Francisco José Francés García, Clemente Penalva Verdú y Óscar Antonio Santacreu Fernández -se les cita alfabéticamente, pues el orden con el que firman las monografías varía de una a otra-.

A pesar de que las áreas de interés de dichos autores abarcan una temática de lo más diverso, todos ellos tienen en común un profundo conocimiento de los aparejos metodológicos y epistemológicos de la investigación social, en forma de las herramientas, técnicas, planteamientos e implicaciones propios de esta. Prueba de ello son no ya esta serie de monografías, sino, en especial, la vasta producción intelectual en forma de libros, artículos y ponencias que todos ellos acumulan a lo largo de sus respectivas carreras -y cuya enumeración resultaría aquí demasiado prolija-, amén de su labor docente. En lo que a esta última se refiere, Clemente Penalva Verdú es profesor de las asignaturas "Análisis de Información Cualitativa”, en el Grado en Sociología, y "Técnicas de Investigación Social en Comunicación", en el Grado en Publicidad y Relaciones Públicas; Antonio Alaminos Chica, catedrático de Sociología Matemática y especialista en técnicas de investigación, análisis y prospectiva electoral, da clases, entre otras materias, de "Análisis de Datos para el Trabajo Social" en el Grado en Trabajo Social; Francisco José Francés García imparte, también entre otras, "Técnicas de Investigación Social para el Trabajo Social" en el Grado de Trabajo Social, "Análisis de Datos para el Trabajo Social" en el Grado en Sociología, "Impactos sobre la Salud y Metodologías de Investigación" en el 
Máster Universitario de Cooperación al Desarrollo, y "Herramientas de Investigación Cuantitativa aplicadas a la Investigación", dentro del Máster Universitario en Comunicación e Industrias Creativas; finalmente, Óscar Antonio Santacreu Fernández tiene a su cargo las asignaturas "Análisis de Datos I" y "Sociología Matemática" en el Grado en Sociología, y "Herramientas de Investigación Cuantitativa aplicadas a la Investigación", en el Máster Universitario en Comunicación e Industrias Creativas.

Hechas estas contextualizaciones preliminares, conviene destacar, por encima de todo, la profunda orientación práctica, incluso instrumental, de La investigación cualitativa... Y ello al menos por tres razones. Primera, por su propósito declarado -al que ya se ha hecho alusión- de servir como referencia durante la impartición de un curso. Segunda, porque, por su estructura y enfoque, se trata claramente de una obra concebida no tanto para ser leída de una manera continuada y unitaria -cosa que, por descontado, puede hacerse sin el más mínimo problema-, sino para instituirse como una guía, fuente de consulta o vademécum al que acudir puntualmente a fin de aclarar conceptos y procedimientos relacionados con la investigación cualitativa. Y tercera, porque, de manera intencionada, el libro elude muchas de las derivaciones filosóficas o epistemológicas a las que podría haberle abocado la materia en cuestión, para centrarse en sus aspectos más pragmáticos, en muchos casos directamente encauzados hacia la actividad investigadora.

El volumen se estructura en tres partes: "La investigación cualitativa", "Análisis cualitativo" y "Análisis cualitativo con Atlas.ti v7". La primera de ellas -en la que es posible rastrear, debidamente aquilatadas, algunas ideas contenidas en manuales clásicos de la disciplina, como los de Valles, Taylor y Bogdan, Ruiz Olabuénaga, Blanchet, Callejo, Ibáñez o Alvira- comienza por abordar los orígenes y desarrollos de las diferencias entre los métodos cuantitativo y cualitativo. Dentro de este terreno, los autores, a pesar de defender la compatibilidad de uno y otro y poner de manifiesto, siguiendo a Wallerstein, el carácter histórico, contingente o no natural de ese antagonismo, avanzan en sus explicaciones mediante la técnica del contraste entre ambos. Surgen así, como conductores argumentativos o ilustrativos, los binomios ciencias naturales/ciencias humanas, positivismo/humanismo (o positivismo/hermenéutica), estudio de los hechos objetivos y externos/de los discursos sociales, formas de acercamiento a la realidad nomotéticas/idiográficas, noción extensiva/intensiva del objeto de estudio, medición/descripción del mismo, esfera de la facticidad/de la cultura simbólica, diseño planificado/emergente, rigidez/flexibilidad, metodología deductiva/inductiva, muestreo probabilístico/no probabilístico (o aleatorio/intencional), encuesta/entrevista en profundidad 
como técnicas paradigmáticas, concepción respondiente (esquema estímulorespuesta)/operante (esquema estímulo discriminativo-respuesta-estímulo reforzador) de la conducta humana (Pérez Fernández, Gutiérrez Domínguez, García García y Gómez Bujedo, 2010), contenido manifiesto/latente, aspectos denotativos/connotativos del lenguaje, objetividad/subjetividad, profesión/oficio, perspectiva distributiva/estructural (Ibáñez, 1896) y El Suicidio (Durkheim)/La ética protestante y el espíritu del capitalismo (Weber) como dos de las obras clásicas más representativas de sendas, en palabras de Beltrán, "vías de acceso a la realidad social" (p. 13).

Siguen a esta especie de campo base teórico algunas notas sobre el diseño en la investigación cualitativa. Un diseño que, evidentemente, debe configurarse en función de los objetivos fijados, y en el que los métodos y las técnicas dependen de muy diversos factores. En ese sentido, por su propio andamiaje interno, la investigación cualitativa rompe con lo que Bourdieu, $\mathrm{Pa}$ sseron y Chamboredon denominan "lógica de la división burocrática del trabajo" (p. 27), que tan bien ejemplifica el célebre círculo de Wallace, y prioriza temporalmente la observación no estructurada de los hechos. También en lo tocante a esta cuestión -el diseño de investigación- se perfilan importantes oposiciones binarias entre la perspectiva cuantitativa y la cualitativa: precodificación/poscodificación, linealidad/circularidad (o forma helicoidal), concreción/abstracción...

En un movimiento muy característico de la obra -como es el desplazamiento desde lo teórico a lo práctico- se despliegan a continuación algunas consideraciones sobre dos de las técnicas preponderantes en el método cualitativo: la entrevista en profundidad y el grupo de discusión o focus group. Completa este bloque un interesante apartado consagrado a las técnicas de observación, con especial énfasis en las directas, esto es, aquellas que tienen lugar "en los escenarios naturales donde se desarrolla la acción social" (p. 58). A su vez, dentro de estas, la atención de los autores se fija, por su carácter esencialmente idiográfico, en la observación participante y en los problemas epistemológicos y metodológicos derivados de esta, en especial la reactividad, los grados de participación, los estadios en que tiene lugar y su propio diseño.

Si la primera parte se enfoca en demarcar las particularidades del método cualitativo (a menudo más visibles por contraste con el cuantitativo, quod erat faciendum) y las técnicas de recolección de datos primarios, la segunda, "Análisis cualitativo", lo hace, como su propio nombre indica, en la fase de evaluación de los datos. Una fase que, debido a su carácter flexible, emergente y disímil, y a la dificultad de compaginar la codificación de los datos con el análisis, reviste más dificultades en su formalización que la del procedimiento cuantitativo. 
No obstante, su práctica puede ser comunicada a partir de dos elementos. El primero es la exposición de la metodología empleada en otros análisis, si es posible desde la idea primigenia hasta los resultados; el segundo es la clasificación de los diferentes modelos detectados a partir de ciertas regularidades procedimentales encontradas en la investigación cualitativa acumulada y que aparecen en diferentes manuales de metodología cualitativa (p. 71).

La mecánica del análisis cualitativo con los datos consta de dos operaciones retroalimentadas: una analítica, de deconstrucción de los mismos, y otra teórica, sintética, de reconstrucción. Para una correcta interpretación de tan sensible material -se nos recuerda- deben sincronizarse cuatro procedimientos: producción/recolección, tratamiento, interpretación y teoría y, a la postre, elaboración del informe final. En este punto, el libro se extiende en una serie de manipulaciones que la mayoría de las veces discurren paralelas al acopio de datos, como son su transcripción, reducción y exposición, entendida esta no como su comunicación o presentación, sino en cuanto acoplamiento y articulación de la información de cara a esbozar conclusiones. A lo largo de toda la etapa resultará fundamental no perder de vista que "la intención del análisis no es describir los datos, sino (...) los objetos o los acontecimientos a los que nuestros datos se refieren" (p. 78).

En el subsecuente pasaje, los autores se aplican en delimitar los tres métodos que, de manera no excluyente, coexisten en el proceder cualitativo: los análisis descriptivos, los generadores de teorías y los basados en el análisis de discurso. En relación a los primeros, se citan varias guías para la exploración del material textual. Una de ellas aconseja atender a los temas, las actitudes, las motivaciones y las atribuciones o designaciones del objeto, a más de a los procesos y las oposiciones que se forjan con respecto a otros entes. Una segunda, que se desarrolla de manera más extensa, tiene que ver con en el esquema circular propuesto por Dey en 1993, sustentado en la descripción (según contextos, intenciones y procesos), la clasificación y el establecimiento de conexiones. Por lo que hace a los análisis generadores de teorías, tras una breve alusión a la inducción analítica -cuya primera formulación apareció en el manual de Znaniecki The Method of Sociology (1934)-, el texto se centra en la Grounded Theory o teoría fundamentada, desplegada por Glaser y Strauss a finales de la década de los sesenta del siglo XX. La enumeración de sistemas de análisis cualitativo se cierra con el análisis de discurso, "un conjunto bastante heterogéneo de métodos y procedimientos basados en la semiótica y en el estructuralismo" (p. 96), que concibe la realidad social como algo construido, en esencia, por el lenguaje. No es de extrañar que, si este último se erige como un elemento clave para acceder al objeto, los instrumentos conceptua- 
les de la lingüística adquieran un protagonismo capital en esta vía. Profundizando en ella, atinadamente se recoge la distinción de Saussure entre lengua (langue) y habla (parole), y se añade otra diferenciación no menos necesaria: la existente entre texto, como materia prima del análisis, y discurso, tal que elaboración teórica realizada sobre dicha materia. Concluye el pasaje con una mención de los tres niveles de análisis propuestos por Ruiz Ruiz (2010) para este tercer método: textual (descriptivo), contextual (comprensivo) y sociológico (explicativo), niveles a los que Fairclough, en Critical Discourse Analysis (1995), se refiere como textual, discursivo y social, y que Alonso sitúa, respectivamente, en torno a los ejes informacional/cuantitativo, estructural/textual y social/hermenéutico en su obra de 1998 La mirada cualitativa en sociología: una aproximación interpretativa.

Las siguientes secciones de la segunda parte se sumergen en las que podrían considerarse fases de salida de la investigación. De esta manera, en la primera de tales secciones, "Interpretación y teoría", y tras señalar dos operaciones que pueden ser simultáneas, como la identificación del significado del discurso (interpretación) y la producción de modelos que recuperen su sentido (análisis), se alude, primero, al papel de la intuición -donde entra en juego el oficio del investigador-y después, a las relaciones entre psicoanálisis y retórica. Una sección posterior se ocupa brevemente de "el control en la investigación cualitativa", para, sin solución de continuidad, dar paso a la última de ellas, dedicada a la exposición de la teoría y la realización del informe correspondiente. En esta etapa crítica cobran especial relevancia la alineación de campos semánticos, la detección de estructuras y, de manera muy singular, el volcado de lo anterior en representaciones gráficas que puedan ilustrar los hallazgos y facilitar su lectura y comprensión.

La tercera y última parte del libro, "Análisis cualitativo con Atlas.ti v7", supone un cambio de tercio radical respecto a las dos anteriores, pues desplaza el foco de atención hacia la utilización y posibilidades de este software concebido para la interpretación de datos textuales (que pueden ser escritos, pero también audiovisuales, por ejemplo, fotografías, sonidos o vídeos). Tras una breve introducción al programa, a sus principales elementos y a su área de trabajo, se pasa a analizar cada uno de los tres niveles de procedimiento empleados en él: textual, conceptual y organizacional. En el primero de ellos, las operaciones básicas consistirían en la fragmentación del texto y su codificación. En el segundo, se trataría de dotar de significado a los elementos instituidos, en un proceso que reproduce, a nivel práctico, el de deconstrucciónreconstrucción realizado a nivel teórico. A tal fin se busca, de inicio, la creación de familias -entendidas como agrupaciones de objetos o componentes según 
ciertos criterios de semejanza- a través del establecimiento de conexiones entre elementos, para, en un nivel posterior (y superior, analíticamente hablando), instituir, en la medida de lo posible, redes entre ellas. Durante el paso de un nivel a otro se recorren todas las posibilidades del programa en cuanto a codificación, funciones de búsqueda y recuperación de texto, generación de familias y redes, formación de vínculos y relaciones (y su visualización), exploración de objetos o la posibilidad de realizar análisis exploratorios, entre otras muchas. En último lugar, se incluye un apartado destinado al análisis de contenido con Atlas.ti. Como es sabido, esta metodología se basa en la precodificación, lo que la convierte en representativa de la vertiente más cuantitativa del análisis textual. Seguramente sea esa la razón por la cual se subsumen en este tramo unas líneas aplicadas a la posibilidad de exportar los datos obtenidos a programas de tratamiento estadístico como el SPSS, con las que se pone punto final al libro.

A la hora de hacer una valoración crítica de La investigación cualitativa... parece de rigor incidir sobre varias cuestiones. La primera de ellas tiene que ver con el innegable dominio de las diversas técnicas de investigación social que muestran los firmantes del volumen. Ese dominio se hace patente no solo en sus propios argumentos, sino también en las muy documentadas fuentes que manejan, entre las que es posible encontrar tanto a las voces más autorizadas en la materia a nivel internacional (Denzin, Dey, Fairclough o Krippendorff, entre otros) como a los más preclaros -y no menos importantes- estudiosos nativos del tema. En relación a estos últimos, merece sin duda una mención especial la omnipresente escuela crítica (Cerrillo Vidal y Ruiz Ruiz, 2012) o "escuela madrileña" (Teira Serrano, 2000, p. 197), de la que formaron parte los malogrados pioneros Jesús Ibáñez o Ángel de Lucas, amén de destacados maestros como Alfonso Ortí, Luis Enrique Alonso o Francisco Pereña.

La segunda cuestión atañe al planteamiento general de todo escrito, que debe tener su correlato ideal en una estructura apropiada. En este caso, puede hablarse de acierto absoluto, al haber trazado los autores un esquema que transita de lo general a lo particular y de lo teórico a lo práctico, cerrando cada vez más el plano de visión, hasta desembocar en la tercera parte, la dedicada al programa Atlas.ti. Es en esta última donde quizá radique una de las mayores fortalezas de la monografía, ya que, a pesar de su brevedad, bien podría complementar, desde un punto de vista metodológico, al manual de referencia en español sobre dicho programa: el ya clásico de Muñoz-Justicia, cuya última versión data de finales de 2015 y se presenta firmada en coautoría junto a Miguel Ángel Sahagún-Padilla. 
La tercera y última cuestión concierne al principal rasero por el que debería ser juzgada cualquier creación, que no es otro que la adecuación entre sus objetivos y sus logros. También aquí se antoja pertinente hablar, y con más razón si cabe, de acierto pleno. Y es que La investigación cualitativa... no aspira, dadas la finalidad para la que ha sido concebida, su corta extensión y su orientación a caballo entre la teoría y la praxis, a erigirse en la magnum opus de este tipo de perspectiva, sino más bien a servir de manera eficaz y sin alharacas al investigador social en aspectos concretos y aplicables de su tarea, propósito que cumple de sobra y a plena satisfacción. A ello contribuyen en gran medida la profusa utilización de figuras -que ilustran e iluminan muchas de las aseveraciones del texto-, un lenguaje tan preciso como accesible y una armazón claramente al servicio de las directrices globales del volumen. En definitiva, una obra si no imprescindible, sí muy recomendable para todos aquellos que deseen iniciarse o afianzar sus conocimientos en los aspectos más funcionales de una metodología como la cualitativa, que en las últimas décadas ha alcanzado, por derecho propio, plena legitimidad en el quehacer científico.

\section{BIBLIOGRAFÍA}

Castro Nogueira, L., Castro Nogueira, M. A. y Morales Navarro, J. (2009). Metodología de las Ciencias Sociales. Una introducción crítica. Madrid: Tecnos.

Cerrillo Vidal, J. A. y Ruiz Ruiz, J. (2012). Ángel de Lucas, maestro y pionero de la sociología cualitativa en España. Revista Internacional de Sociología, 70(3), 697700. Recuperado de http://revintsociologia.revistas.csic.es/index.php/revintsocio logia/article/view/479/500.

Colás-Bravo, P. (1987-88). La metodología cualitativa en el estudio de cuestiones educativas. Cuestiones pedagógicas: Revista de ciencias de la educación, 4-5, 79-90. Recuperado de http://institucional.us.es/revistas/cuestiones/4_5/art_7.pdf

Ibáñez, J. (1986). Perspectivas de la investigación social: el diseño en las tres perspectivas. En M. García Ferrando, J. Ibáñez y F. Alvira (Comp.), El análisis de la realidad social. Métodos y técnicas de investigación social (pp. 57-98). Madrid: Alianza.

Muñoz-Justicia, J. y Sahagún-Padilla, M. A. (2015). Hacer análisis cualitativo con ATLAS.ti 7: Manual de uso. http://dx.doi.org/10.13140/RG.2.1.4837.6405

Pérez Fernández, V. J., Gutiérrez Domínguez, M. J., García García, A. y Gómez Bujedo, J. (2010). Procesos psicológicos básicos: un análisis funcional. Madrid: UNED.

Ruiz Ruiz, J. (2010). Análisis sociológico del discurso: métodos y lógicas. Forum Qualitative Sozialforschung / Forum: Qualitative Social Research, 10(2), Art. 26. Recuperado de http://www.qualitative-research.net/index.php/fqs/article/view/ $1298 / 2776$

Teira Serrano, D. (2000). Reseña de "La mirada cualitativa en sociología", de Luis Enrique Alonso. Reis. Revista Española de Investigaciones Sociológicas, 91, 196-199. Recuperado de http://www.reis.cis.es/REIS/PDF/REIS_091_11.pdf. 
AGUSTÍN GARCÍA-RAMOS es graduado en Sociología por la UNED y la Universidad de Alicante, en cuyo Departamento de Sociología II disfrutó de una Beca de Colaboración del Ministerio de Educación, Cultura y Deporte durante el curso 2015-16. Además, posee un Máster Ejecutivo en Community Management y Dirección de Redes Sociales en la Empresa por la propia Universidad de Alicante. Actualmente colabora en el proyecto "La participación política como candidatos de los residentes europeos en España", desarrollado por el Observatorio Europeo de Tendencias Sociales (OBETS). 